\title{
Ventilator-Associated Pneumonia Caused by Klebsiella Pneumo- niae in Preterm Newborn Infants
}

\author{
Kushnareva MV*, Markhulia Kh M, Keshishyan ES and Semenov AV \\ Veltishchev Research Clinical Institute of Pediatrics, Russian National Research Medical Pirogov's \\ University, Ministry of Health of the Russian Federation, Russia
}

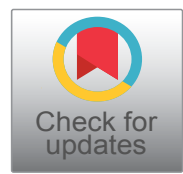

*Corresponding author: Kushnareva MV, Veltishchev Research Clinical Institute of Pediatrics, Russian National Research Medical Pirogov's University, Ministry of Health of the Russian Federation, Russia, Tel: +74991574457, E-mail: mkuschnareva@mail.ru

\begin{abstract}
Background: The basis for the study is the high morbidity and mortality in premature infants with ventilator-associated pneumonia (VAP). There is a high incidence of complications (sepsis, bronchopulmonary dysplasia).

Methods: A detailed analysis of the clinical course of VAP caused by Klebsiella pneumoniae in 14 premature infants was conducted. The sensitivity of Klebsiella pneumoniae strains to 18 antibiotics was studied by the method of standard discs.

Results: Severe pneumonia had 10 infants, moderate stage was among 4 infants, an acute stage was observed in 12 patients, and prolonged stage was in 2 infants. Severe form of the disease was presented by pronounced symptoms of infectious toxicosis, respiratory and cardiovascular insufficiency, physical changes from the lungs. Bronchopulmonary dysplasia was developed in two infants. The mortality was rated $14 \%$. Klebsiella pneumoniae was the causative agent of the primary infection in all infants (monoinfection was in 12 infants and association with other pathogens in 2 infants). All strains of Klebsiella pneumoniae were multidrug-resistant to antibiotics, but $57-86 \%$ of the strains retained sensitivity to Piperacillin, Piperacillin + Tazobactam, carbapenems, Amikacin and Ofloxacin.
\end{abstract}

Conclusions: The complex treatment of VAP in premature infants should include antibacterial, detoxification, pathogenetic and syndromic therapies.

\section{Keywords}

Preterm newborn infants, Ventilator-associated pneumonia, Clinical course, Klebsiella pneumoniae, Antibiotics

\section{Introduction}

The modern neonatology made considerable progress in nursing and treating of premature newborn infants. However, the problem of nosocomial ventilator-associated pneumonia (VAP) in this category of patients is still relevant [1-3]. This disease is accounted for $20 \%$ of nosocomial infections [1]. It may cause death or development of severe chronic pathology of the respiratory tract [1-3]. Although many scientific studies are devoted to the problem of ventilator-associated pneumonia (VAP) in premature newborn infants [1-6] however, there are few works concerned with the study of the clinical course of the disease $[3,7,8]$.

During the last 10 years, the infection is still relevant, including VAP in preterm newborn infants which is caused by Klebsiella pneumoniae ( $K$. pneumoniae) $[5,6,9]$. The epidemiological importance of this pathogen is largely owing to its biological properties, specifically, the formation and proliferation of new hospital strains, polyresistance to antibiotics, the ability to remain for a long time on surfaces of the objects in environment, including medical equipment parts $[9,10]$. The multiresistant strains of $K$. pneumoniae are particularly dangerous. There are two fatal cases of severe infection (pneumonia, abscess, sepsis) among patients. The disease was caused by the unique hospital strain of $K$. pneumoniae. This isolate had a unique resistance to antibacterial drugs (sensitive to one from 30 tested antibiotics only) [11].

Citation: Kushnareva MV, Markhulia Kh M, Keshishyan ES, Semenov AV (2018) Ventilator-Associated Pneumonia Caused by Klebsiella Pneumoniae in Preterm Newborn Infants. Int J Pediatr Res 4:031. doi. org/10.23937/2469-5769/1510031

Received: May 05, 2017: Accepted: March 17, 2018: Published: March 19, 2018

Copyright: (C) 2018 Kushnareva MV, et al. This is an open-access article distributed under the terms of the Creative Commons Attribution License, which permits unrestricted use, distribution, and reproduction in any medium, provided the original author and source are credited. 
In our opinion, it is important to make a detailed analysis of the clinical course of VAP, caused by $K$. pneumoniae in a large group of patients and study of the biological properties of pathogens. This will allow to determine optimal treatment VAP in preterm infants and successfully fight nosocomial infections in Intensive care unit. It will allow to determine an optimal treatment VAP in preterm newborn infants and successfully fight with nosocomial infections in resuscitation and intensive care unit.

\section{Objective}

To research the clinical features of ventilator-associated pneumonia caused by $K$. pneumoniae in premature newborn infants and properties of strains-pathogens.

\section{Materials and Methods}

We observed 14 preterm newborn infants with VAP caused by $K$. pneumoniae as monoinfection in 12 infants and mixed infection in 2 infants. Body weight at birth varied from 840 to $2750 \mathrm{~g}(18315 \pm 170.01 \mathrm{~g})$ and gestational age was from 27 to 37 weeks (32.92 \pm 0.925 weeks). Among them there were 6 girls and 8 boys. The study was conducted January 2013 till December 2014.

The microbiological examination of tracheobronchial aspirates (TBA) and oral swabs from the back of the throat was examined in all infants. This examination was performed by standard quantitative method for all infants for a wide range of nutrient media for the isolation of aerobic and facultative organisms [12]. The study was conducted at onset of VAP and days 2-3 times in the course of the disease after 7-14. All bacterial cultures were identified by commercial test systems API $20 \mathrm{NE}$ "BioMerieux» (France), E | NE "Crystal" (USA), NEFERM test "Lachema" (Czech Republic) in accordance with the manufacturer's instructions. The number of microorganisms was expressed in the following units:

1. The number of colony forming units in 1 standard tampon - in oral swab cultures (CFU/st) or biofluid in $1 \mathrm{ml}(\mathrm{CFU} / \mathrm{ml})$ in microbial contamination up to 1000 cells.

2. The decimal logarithm ( $\mathrm{g}$ ) in microbial load 1000 and more microbial cell in 1 tampon or $1 \mathrm{ml}$ of biofluid. Etiologically significant number of microbial cells believed Ig4 and higher CFU/ml for TBA, Ig6 and higher CFU/t for the smear with mucous posterior pharyngeal [13].

Sensitivity determination of isolated strains bacteria (K. pneumoniae and bacteria - Assiociants) antibiotic conducted disk-diffusion method on agar Mueller-Hinton (Mueller Hinton Agar) using standard commercial drive test systems NITSF (Russia) and test systems ATV pse 5 (BioMerieux) [14].

\section{Results}

\section{Maternal health}

All the children were born prematurely in women with a history of physical and obstetric-gynecologic anamnesis. There were 5 primipara mother, and 9 multipara mothers. Chronic somatic diseases such as (asthma, bronchitis, pyelonephritis, gastritis, and others) were observed in $50 \%$ of women, and chronic obstetric-gynecologic diseases (habitual miscarriage, infertility, adnexitis, cervical erosion, colpitis, uterine fibroids) occurred in $64 \%$. Pregnancy complications as toxicosis in the $1^{\text {st }}$ half were observed in $29 \%$ of women, threatening miscarriage in $43 \%$, acute respiratory viral infection - in $28 \%$ of women.

As a whole 10 mothers (71\%) had spontaneous labor and 4 women (23\%) had operative delivery. Accelerated labor was met in 3 women (9\%). Foot or pelvic presentations were met in 2 cases (14\%). Birth complications in the form of xerotocia were in 3 patients $(21 \%)$, placental abruption and bleeding during childbirth was in 13 women (7\%). Chorioamnionitis was diagnosed in 1 women (7\%). Meconium in the water was in 1 women (7\%).

\section{The clinical course of VAP}

The condition of all infants at birth was critical. All the children were born in asphyxia, including severe condition in 12 newborns. Severity of the condition at birth caused by the presence of respiratory, cardiovascular inefficiency and changes in the central nervous system (CNS). Indications for artificial pulmonary ventilation were respiratory disorders. They were caused by respiratory distress-syndrome (RDS) in 12 patients $(86 \%)$, aspiration syndrome amniotic fluid in 2 patients (14\%). Artificial lung ventilation (ALV) was notices in 12 infants at birth (86\%), ALV with 1.5 till 14 hours frequency was in 1 infants (14\%). Duration of AVL was $5.49 \pm$ 1.56 days in average.

Pneumonia was diagnosed on the 4-12 days of life (on the 4-12 days from the beginning of ALV). A severe form of pneumonia was in 10 infants (71\%), medium clinical course was in 4 patients (29\%), acute course was observed in 12 patients (86\%), prolonged duration, i.e. disease duration for more than 50 days was in 2 patients (14\%).

The beginning of pneumonia among the majority of infants refers to the first week of life (in 12 infants). The clinical picture was shown by onset of initial symptoms of infectious toxicosis by increasing respiratory failure, the growth and change in the nature of physical changes in the lungs. ALV parameters become insufficient for adequate ventilation. Ordinary, especially in infants with weight $\leq 1500 \mathrm{~g}$, during this period it was hearing a systolic heart murmur; there was a pulsation of the arteries, a tendency to tachycardia, indicating that the shunting of blood through the patent ductus arteriosus.

The majority of infants have a severe form of the disease which was developed florid symptoms of infectious toxicosis, respiratory failure and physical changes 
of the lung. Also the great initial weight loss pointed out on the severity of the condition and an unfavorable course of adaptation processes (more than $12 \%-17 \%$ in 10 infants).

The changes in the lungs have always been two-sided and characterized by the presence of shortcut resonance, large, wet widespread and crepitant rattling in the lungs. Symptoms of tracheobronchitis with duration from 8 till 26 days were observed in 6 infants (46\%).

The X-ray determined a double pulmonary involvement in most of cases (in 12 infants) in the form of macro focal areas of pneumatization reduction, strengthen bronchovascular drawing. Hydropic changes (segmental or general) along with focal shadows were determined in 7 infants. It was determined lobar atelectasis in 2 infants.

The changes in CNS stood out in the form of significant suppression of physiological reflexes, including those stable as sucking and nasopalpebral, motion activity and muscle tonus in 10 infants. Convulsive readiness and convulsion was observed only in 1 infant.

Clinical manifestation of infection in acute toxicosis VAP characterized as hypothermia- in 5 infants (38\%), single or short-term increase $t^{\circ}$ of body within 37.5-38.0 ${ }^{\circ} \mathrm{C}$ in 9 infants (64\%). Grey color of the skin cover was in 6 infants (43\%), «marbling» - in 4 (29\%), hepatomegaly in $4(29 \%)$, splenomegaly $2(14 \%)$.

Changes in the respiratory system in the acute period VAP characterized by the presence of localized cyanosis in 13 infants (93\%). Diffuse cyanosis was only in 1 infant (7\%). Rapid breathing up to $51-80$ per minute was in all infants.

Increased rapidity of the chest was observed in 3 infants $(21 \%)$, shortening of percussion sound was in 14 (100\%). Permanent heavy crackles in the lungs listened in 13 infants (94\%), and dry rale only in 1 (7\%). Pulmonary hemorrhage was diagnosed in 1 infant (7\%).

The index of acid-base balance of blood indicated to the presence of metabolic acidosis in 3 infants (21\%), respiratory acidosis in 4 (29\%), mixed acidosis in $3(21 \%)$ infants. The average $\mathrm{pCO}_{2}$ in the blood was $62.2 \pm 3.40$ $\mathrm{mmHg}$ and $\mathrm{pO}_{2}$ in blood $41.4 \pm 2.12(\mathrm{mmHg})$. The oxygen-function blood was distressed. The level of $\mathrm{Hb}$ was lowered in 7 infants in average up to $100.6 \pm 7.02 \mathrm{~g} / \mathrm{l}$.

There were changes in the cardiovascular system (CVS) in the form of weakening heart tones in 4 infants, an increase of its boundaries, the development of edema, tachycardia in 3 infants and bradycardia in 1 infants.

The inflammatory process in the lung was combined with pathological symptoms from other organs and changes of laboratory parameters. In the peripheral blood during the acute period VAP was observed leukocytosis in 7 infants (50\%) with increase of the number of white blood cells to $32.8 \cdot 10^{9} \pm 6.69 /$ l. Neutrocytosis was in 5 infants (36\%). The toxic granularity of neutrophils was not detected. Thrombocytopenia was occurred in 2 infants (14\%).

Changes in blood biochemical values were as follows: Hypoproteinemia (protein content of $<55 \mathrm{~g} / \mathrm{l}$ ) was in 3 infants (21\%). 3 infants had an increased content of transaminases, including AST $\geq 50 \mathrm{mkmol} / \mathrm{ml}$ was in 2 infants (14\%), ALT was $\geq 50 \mathrm{mkmol} / \mathrm{ml}$ in 3 infants (21\%), decrease of Na was in $2(14 \%)$. The level of C-reactive protein was raised in 2 infants and was 20.0 and $22.2 \mathrm{mg} /$ liter at the rate up to $6 \mathrm{mg} / \mathrm{l}$.

It was revealed that 2 infants had renal dysfunction in the form of reduced diuresis in the first days of illness $(<2.1 \mathrm{ml} / \mathrm{kg} /$ hour), increasing of urea level $\geq 7 \mathrm{mmol} / \mathrm{l}$ in 8 infants in the serum blood. Edematous syndrome was observed in 9 newborns. Pathological changes were observed in the general analysis of urine, in the form of proteinuria - in 7 infants, leukocyturia - in 6, microhematuria - in 8, bacteriuria - in 1, Candiduria - in 1 infant.

The duration of pneumonia survivors was from 28 to 65 days and compound in average $(\mathrm{M} \pm \mathrm{m}) 42.0 \pm 3.95$ days. Severe condition lasted about $28.7 \pm 3.05$ days, including the phenomena of infectious toxicosis -22.3 \pm 0.58 days. Respiratory failure was observed from 13 to 37 days ( $24.8 \pm 5.24$ days), rattling in the lungs was heard $22.0 \pm 3.82$ days. Staying in an oxygen hood was $16.6 \pm 5.11$ days. The necessity of infants staying in an incubator was $30.0 \pm 5.64$ days and the necessity of tube feeding was $28.08 \pm 4.01$ days. The positive dynamics of body weight was noted in average with $17.8 \pm 1.79$ days of life. Stationary admission time of infants in a hospital was $42.5 \pm 3.42$ bed days. As VAP complications was diagnosed bronchopulmonary dysplasia in 2 infants (14\%). 2 infants were died (14\%) with a birth weight less than $1000 \mathrm{~g}$.

Five infants with the change in VAP pathogen was noted longer duration of the disease (from 50 to 65 days) in comparison with infants without a change of pathogen (from 28 to 44 days). Severe condition and infectious toxicosis were prolonged (for 6-12 days), and the number of the antibiotic therapy' courses was for 1-3 more when the pathogen was changed.

The clinical indices were not different from the mean values for the monoinfection of $K$. pneumoniae in 2 infants with initial mixed infection.

We compared the clinical course of VAP, associated with $K$. pneumoniae, with the VAP clinic with a different etiology that was described in previous studies $[15,16]$. Thus, the mixed infection was 2 times less frequent met and the secondary infection was 1.8 times less frequent met in infants with VAP, associated with $K$. pneumoniae. Moreover, the mortality was 2 times lower in infants with VAP, associated with $K$. pneumoniae than in infants with VAP caused by Pseudomonas aeruginosa (P. aeruginosa) [15]. In infants with VAP due to $K$. pneu- 
Table 1: The sensitivity of clinical strains Klebsiella pneumoniae to antibiotics $(n=14)$.

\begin{tabular}{|l|l|l|l|l|}
\hline & Antibiotics & S & R & SR \\
\hline 1 & Azlocillin & 0 & 14 & 0 \\
\hline 2 & Meropenem & 10 & 2 & 2 \\
\hline 3 & Imipenem/Cilastatin & 10 & 2 & 2 \\
\hline 4 & Piperacillin & 10 & 0 & 4 \\
\hline 5 & Piperacillin + Tasobactam & 12 & 0 & 2 \\
\hline 6 & Carbenicillin & 0 & 14 & 0 \\
\hline 7 & Cefotaxime & 0 & 14 & 0 \\
\hline 8 & Ceftazidime & 0 & 14 & 0 \\
\hline 9 & Ceftriaxone & 0 & 14 & 0 \\
\hline 10 & Cefoperazone & 2 & 8 & 4 \\
\hline 11 & Cefaclor & 2 & 12 & 0 \\
\hline 12 & Amicacin & 6 & 6 & 2 \\
\hline 13 & Gentamicin & 2 & 12 & 0 \\
\hline 14 & Kanamycin & 1 & 11 & 2 \\
\hline 15 & Ofloxacin & 10 & 2 & 2 \\
\hline 16 & Ciprofloxacin & 4 & 10 & 0 \\
\hline 17 & Chloramphenicol & 6 & 8 & 0 \\
\hline 18 & Tobramicin & 8 & 4 & 2 \\
\hline
\end{tabular}

S: Sensitive stain; R: Resistant stain; SR: Stain with intermediate sensitivity.

moniae, BPD was 2 times less developed, weight gain was stabilized 12 days earlier and mixed infection was twice less frequent, compared to VAP, caused by Stenotrophomonas maltophilia (Sten. maltophilia). However, there was no lethal in the presence of VAP, associated with Sten. maltophilia [16]. Sepsis was developed with infection caused by $P$. aeruginosa (in $6 \%$ of infants) and Sten. maltophilia (13\%). In addition, there was a tendency to a longer period of toxicosis ( 5 and 6 days in average, respectively) and a severe condition of the patients ( 4 and 5 days in average, respectively), and renal dysfunction (in 29 and $67 \%$ infants, respectively) with these VAP pathogens $[15,16]$. Hemorrhagic syndrome was developed in $29 \%$ of infants in VAP, caused by $P$. aeruginosa [15]. Thus, the course of VAP, caused by $K$. pneumoniae in most infants was less severe than in the other etiology of the disease.

\section{Microbiological studies}

There were identified strains of $K$. pneumoniae among all the surveyed infants. It should be noted that $K$. pneumoniae as a pathogen of a primary infection was in 14 infants including as monoinfection in 12 infants and in association with other opportunistic pathogen in 2 infants. In conjunction with $K$. pneumoniae there were found $E$. coli in one infant, Enterococcus faecalis (Ent. faecalis) in one infant. All these microorganisms were present in TBA in etiologically significant quantities.

There was a single change of the pathogen in 4 newborns $(28 \%)$ among them. In the primary isolation there were marked $K$. pneumoniae.

After $8.0 \pm 0.83$ days (with surge from 6 to 12 days) after the first survey was released in the etiologically significant amount of Pseudomonas aeruginosa ( $P$. aeruginosa) by 2 infants and $E$. coli by 2 infants. It was accompanied by a deterioration of the clinical course of the disease. The change of pathogen was occurred twice in 1 infant. $K$. pneumoniae was allocated in the primary isolation; $P$. aeruginosa was allocated after 7 days and Ent. faecalis was allocated after next 11 days.

The study of sensitivity to antibiotics of other 14 clinical strains of $K$. pneumoniae is shown in Table 1.

All 14 clinical K. pneumoniae strains had individual antibiogram. The most strains (57-86\%) saved sensitivity to piperatsilinu, piperacillin/tazobactam, carbapenems and ofloxacin. Thus all strains were multiresistant to a different spectrum of antibiotics.

E. coli strains were sensitive to amikacin and $\beta$ - lactam antibiotics pluripotent. Ent. faecalis strain was sensitive to vancomycin, linezolid and imipenem/cilastatin. $P$. aeruginosa strains were sensitive to amikacin, piperacillin and piperacillin + tazobactam.

Hemocultures were sterile in all babies. VAP pathogens were found in the oral swab cultures in all infants, and their number was 10-100 times higher than it was in TBA. A concomitant microflora (Stapylococcus epidermidis, Streptococcus viridans, Neisseria sicca) was detected in the oral swab cultures of 7 infants in a small amount (no more than $250 \mathrm{CFU} / \mathrm{st}$ ). These microorganisms were not detected in TBA.

\section{VAP treatment}

Etiological treatment was conducted with sensitivity pathogens to antibiotics. Infants prescribed piperacillin, piperacillin + tazobactam, amikacin, carbapenems alone or in combination with amikacin. If there gram positive coccus (Ent. faecalis) administered vancomycin. Antibacterial therapy was combined with the appointment of immunoglobulin preparations for intravenous administration (Octagam, Pentaglobin or Intraglobin) during 3-5 days. All the infants also received pathogenetic therapy. Treatment of respiratory failure was carried out by the usage of mechanical artificial ventilation, then through a nasal catheter, mask and tent. The duration of ventilator apparatus was from 4 to 18 days.

Newborns also received infusion therapy. According to the testimony for the treatment of cardiovascular insufficiency digoxin maintenance dose every 12 hours and korglikon were ordered to infants. In case of hypovolemia and hypotension were used colloids and/or vasoactive drugs (dopamine and dobutamine).

Infants received probiotics with Bifidobacterium spp. for the prevention and treatment of dysbiotic disorders in the gut.

\section{Discussion}

Emergence of VAP in infants preceded RDS, aspiration syndrome amniotic fluid, syndrome of early postnatal adaptation of breath, cardiovascular and CNS. The development of the disease was occurred in AVL con- 
ditions with high oxygen concentration and inspiratory pressure. The pathogenic micro-organisms actively penetrate in the lower respiratory tract from the external environment and from the upper respiratory tract of a newborn. The endotracheal tube into the airway off a significant portion of the local immune protection of the mucous membrane $[1,3,4,7]$.

Strains of $K$. pneumoniae have pronounced toxic properties against the infant's body. Thus, bacterial proteases have destructive effects on the respiratory tract tissues and other organs. They contribute to the invasion of the pathogen in the blood and the spread of infection $[6,10]$. Studied strains have a very strong ability to produce mucus. $[6,17]$. They also exhibit hemolytic activity, high adhesive activity, studied on the model of native human erythrocytes. Studied strains produce DNA-ase. They are resistant to the most commonly used classes of antibiotic possess $\beta$-lactamase spread spectrum. Klebsiella strain (Klebsiella pneumonia subsp. Pneumoniae) - t produce $\beta$-lactamase -class CTXM 1 [17].

It is known the immunosuppressive effect of $K$. pneumoniae on lymphocytes of infants. The apoptosis of lymphocytes was examined in 10 infants with late neonatal sepsis induced by $K$. pneumoniae 7 infants were preterm. Acute phase of neonatal sepsis is associated with an increased apoptosis of peripheral blood lymphocytes [6].

The biological properties of bacterial pathogens influence the clinical manifestations of the disease.

It is proceed with a serious infectious toxicity, longterm respiratory and cardiovascular insufficiency, physical changes in the lungs, severe depression of the central nervous system, peripheral circulatory disorders, development of bronchopulmonary dysplasia.

\section{Conclusions}

Thus, VAP caused by $K$. pneumoniae has acute and severe course in most of preterm infants. The disease occurs with acute toxicosis, severe respiratory failure, widespread bilateral lung and bronchial damage, cardiovascular, and CNS disorders. The complications in the form of atelectasis and bronchopulmonary dysplasia are rarely occurred. The mortality is rated $14 \%$.

Most of infants VAP have monoinfection. Secondary infection is rarely met.

All strains of $K$. pneumoniae are multiresistant to antibiotics, but $57-86 \%$ of strains remain sensitive to Piperacillin, Piperacillin + Tazobactam, carbapenems, Amikacin and Ofloxacin.

Complex treatment of VAP in premature newborns should include antibacterial, detoxification, pathogenetic and syndromic therapies.

\section{Acknowledgement}

We thank the translator Ivleva Elena.

\section{References}

1. Cernada M, Brugada M, Golombek S, Vento M (2014) Ventilator-Associated Pneumonia in Neonatal Patients: An Update. Neonatology 105: 98-107.

2. Camacho-Gonzales A, Spearman PW, Stoll J (2013) Neonatal infectious diseases: evaluation of neonatal sepsis. Pediatr Clin North Am 60: 367-389.

3. Yuan TM, Chen LH, Yu HM (2007) Risk factors and outcomes for ventilator-associated pneumonia in neonatal intensive care unit patients. J Perinat Med 35: 334-338.

4. Cernada M, Aguar M, Brugada M, Gutierrez A, Lopez JL, et al. (2013) Ventilator-associated pneumonia in newborn infants diagnosed with an invasive bronchoalveolar lavage technique: a prospective observational study. Pediatr Crit Care Med 14: 55-61.

5. Chistyakova GN, Sharipova BT, Tarasova MN, Bilimova SI, Gasieva IA, et al. (2012) Features of clinical and immunological adaptation, the nature of microbial colonization in infants with extremely low body weight. Uralskiy Meditsinskiy Zhurnal 11: 22.

6. Khaertynov KhS, Boichuk SV, Anokhin VA, Ramazanov BR, Rizvanov AA, et al. (2015) Lymphocyte apoptosis in neonatal sepsis due to Klebsiella. Geni i Kletki 10: 106-109.

7. Apisarnthanarak A, Holzman Pazgar G, Hamvas A, Olsen MA, Fraser VJ (2003) Ventilator- associated pneumonia in extremely preterm neonates in neonatal intensive care unit: characteristics, risk factors and outcomes. Pediatrics 112: 1203-1289.

8. Xu XF, Ma XL, Chen Z, Shi LP, Du LZ (2010) Clinical characteristics of nosocomial infections in neonatal intensive care unit in eastern China. J Perinat Med 38: 431-437.

9. Bukharova EV, Popkova SM, Rakova EB, Dzhioev YuP, Shabanova NM, et al. (2013) Microecolocical characteristics of Klebsiella spp. Regional Populations in the Intestinal Microbiota of Irkutsk Children 90: 93-96.

10. Shaginyan IA (2000) Role and Significance of Molecular Methods in Epidemiological Analysis. CMAC 2: 82-95.

11. Maltezou HC, Kontopidou F, Katerelos P, Daikos G, Roilides E, et al. (2013) Infections caused by carbapenem-resistant Gram-negative pathogens in hospitalized children. Pediatr Infect Dis J 32: 151-154.

12. Kogan GU, Gimninova IS, Karnoukhova OV, Botvinkin AD, Reshetnikova UV, et al. (2010) Lethal nosocomial infection associated with multiresistent Klebsiella pneumoniae. Siberian Medical Journal 99: 156-158.

13. (1985) On the harmonization of microbiological (bacteriological) research methods used in clinical laboratories of medical institutions. Russia, 126.

14. (2004) Methods of control: Biological and microbiological factors. The definition of sensitivity of microorganisms to antibacterial preparations. Methodical instructions.

15. Kushnareva MV, Markhulia KM, Dementyeva GM, Keshishian ES, Shaginyan IA, et al. (2017) Ventilator-associated pneumonia caused by Pseudomonas aeruginosa in preterm newborn infants. ES Paediatrics 3.4: 390-398.

16. Kushnareva MV, Markhulia KM, Dementyeva GM, Chursina ES, Keshishian ES, et al. (2017) Hospital-acquired pneumonia associated with the causative agent Stenotrophomonas maltophilia, in newborn infants. Russian Bulletin of Perinatology and Pediatrics 62: 53-58.

17. Smirnova L, Zabrovskaya A, Egorova S, Prikhodko E, Yarikova V, et al. (2014) Biological properties of microorganisms species of Klebsiella pneumoniae subsp. pneumoniae isolated from milk cows mastitis. International Veterinary Journal of Veterinary Medicine 2: 12-16. 